\title{
Hardening in thermally-aged Fe-Cr binary alloys: Statistical parameters of atomistic configuration
}

\author{
Tomoaki Suzudo ${ }^{\mathrm{a}, *}$, Yasuyoshi Nagai ${ }^{\mathrm{b}}$, Daniel Schwen ${ }^{\mathrm{c}, 1}$, Alfredo Caro ${ }^{\mathrm{c}}$ \\ ${ }^{a}$ Center for Computational Science and e-Systems, Japan Atomic Energy Agency 2-4 Shirane Shirakata \\ Tokai-mura, 319-1195, Japan \\ ${ }^{b}$ The Oarai Center, Institute for Materials Research, Tohoku University, 2145-2 Narita-cho, Oarai, Ibaraki \\ 311-1313, Japan \\ ${ }^{c}$ Materials Science and Technology Division, Los Alamos National Laboratory, P.O. Box 1663, Los \\ Alamos, NM 87545, United States
}

\begin{abstract}
By exploiting Monte Carlo methodology and molecular dynamics, we computationally simulate the spinodal decomposition of iron-chromium binary alloys and analyze the relationship between the increase of yield strength induced by the phase separation phenomenon, and statistical parameters of the atomistic configuration. We successfully model the experimentallydiscovered proportional relationship between the hardness and the variation parameter (or $\mathrm{V}$ ), and also found that the adequacy of the parameter $\mathrm{V}$ as an empirical indicator of hardening is limited, because it does not properly capture short-range atomistic configurations that influence the hardening. We suggest that the short-range-order parameter has more potential to become universal descriptor of the phenomenon.
\end{abstract}

Keywords: spinodal decomposition, iron-chromium alloy, thermal aging, hardening, molecular dynamics, Monte Carlo simulations

\section{Introduction}

Iron-chromium (Fe-Cr) binary alloys are model alloys for fully ferritic steels and the ferrite phase of cast stainless steels. It is widely known that such alloys with Cr concentration more than $\sim 20 a t . \%$ undergo spinodal decomposition when they are thermally aged because of the miscibility gap [1-3]. This microstructural evolution causes hardening, and loss of ductility, of the material [4,5], and this degradation of mechanical properties is of critical engineering concern because these kinds of steels are ubiquitous as structural materials in nuclear engineering [6]. The mechanism of this phenomenon has long been studied theoretically $[7,8]$ and experimentally $[9,10]$.

\footnotetext{
${ }^{*}$ Corresponding author. Tel.: +8129282 5434

Email address: suzudo.tomoaki@jaea.go.jp (Tomoaki Suzudo)

${ }^{1}$ Present address: Fuel Modeling and Simulation, Idaho National Laboratory, P.O. Box 1625, Idaho Falls, ID 83415-3840, United States

Preprint submitted to Acta Materialia

February 12, 2015
} 
Thanks to atom probe tomography devices, it is possible to directly observe the development of this phase separation phenomenon with nanometer scale, and we can intuitively understand that the cause of the hardening is the interaction between dislocations and the spinodally modulated structure, i.e., Cr-rich (or $\left.\alpha^{\prime}\right)$ phase. Except at low temperatures, screw dislocations can bypass the $\alpha$ '-phases by cross-slip, while edge dislocations remain on the same glide plane and thus cannot bypass the $\alpha^{\prime}$-phases; edge dislocations are expected to be a major cause of hardening [11] at the temperatures where the steels are actually used.

Although many theoretical studies contribute to the modeling of interaction between edge dislocations and Cr-precipitates [11-13], modeling studies for the hardening caused by the spinodal decomposition are limited to simplified modeling, such as [8]. This is probably because dislocation motions in heterogeneous media, such as spinodally decomposed structures, are extremely complicated, and quantitative prediction of such hardening is unrealistic. A realistic approach to this may be to correlate the hardening to statistical parameters of the atomistic configuration of Fe-Cr systems; such parameters may be an amplitude and a wavelength of the modulation pattern. Once a universal relationship between these parameters and the hardening becomes established, the hardening could be monitored without mechanical testings, in which statistical accuracy cannot be obtained easily.

The variation parameter (or V) [14] is also discussed as such a characteristic parameter; this is defined as the integral of the difference between the solute concentration distribution measured from the sample material and the corresponding binomial distribution. Pareige et al. [15] experimentally show that the increase in hardness is linearly related to $\mathrm{V}$. This result importantly suggests that the hardening led by spinodal decomposition can be predicted if one can predict or measure the development of $\mathrm{V}$, and recent related papers [16-18] seem to use the $\mathrm{V}$ as a standard indicator of this hardening development. However, the physical meaning of this linear relationship is still unclear. Besides, we need to know whether the $\mathrm{V}$ parameter is the best to describe the hardening compared with other characteristic parameters, such as an amplitude and a wavelength of the modulation pattern, and a shortrange-order (SRO) parameter [19].

Such a comprehensive study would be rather cumbersome with experimental means, but computer simulation techniques using interatomic potentials can address such a formidable task. For example, kinetics of a dislocation interacting with obstacles is widely used to study hardening caused by precipitates, voids, bubbles, and interstitial loops. By exploiting such atomistic analysis one can gain access to information that is not easily available in analytical or experimental studies.

In the present paper, we use computer simulations to first measure these parameters at various stages of the spinodal decomposition and attempt to find the physical meaning of the $\mathrm{V}$ from inter-parameter analyses. Second, we thoroughly investigate the relations between the hardening and all the parameters characterizing the microstructure. For this purpose, we numerically simulate an edge dislocation moving through the spinodally modulated structure by applying shear strain and monitored the stress tensor, which clear indicates the onset of plasticity or yield point. We successfully observe the experimentally-discovered proportionality between the hardness, that we relate to the yield strength we measure, and V, but we also observe that the universality of $\mathrm{V}$ as an indicator of hardening has a limitation as this 
parameter does not seem to properly capture the short-range atomistic configurations that influence hardening. Comparative studies suggest that the short-range-order parameter has a possibility to become a more universal indicator of hardening.

\section{Computational methodology}

An atomic block of body-centered-cubic (bcc) Fe crystal is created with $25 \%$ atomic concentration of Cr, randomly distributed. See Table 1 for the dimensions of the block and Fig. 1 for crystallographic orientation. Periodic boundary conditions for $x$ and $y$ directions, and the free boundary condition for the $z$ direction are used. As seen in the figure, an edge dislocation along the $y$ direction, i.e. $\langle 11 \overline{2}>$ direction, was inserted into the atomic block. Then, the atomic positions and the system volume were relaxed; the fully relaxed atomic block is the initial state for the numerical simulation of spinodal decomposition. Up-to-date Fe-Cr empirical potentials are the two-band model [20] and the concentrationdependent embedded atom method [21, 22], and Bonny et al. [23] gives a comparative study that concluded that these two models are equivalent approaches. Throughout this study, the concentration-dependent embedded atom method for $\mathrm{Fe}-\mathrm{Cr}$ is used together with the molecular dynamics platform LAMMPS [24].

To numerically simulate thermal aging, we use the variance-constrained semi-grandcanonical (VC-SGC) Monte Carlo algorithm [25]. In this method, the system is evolved by a combination of Monte Carlo chemical swaps, alternated by a number of molecular dynamics steps. The method allows to keep the solute concentration close to a desired set point within the miscibility gap, via a proper choice of the variance parameter. We model three cases by varying the temperature in the Monte Carlo simulation $\left(T_{M C}\right)$ with temperatures of $273^{\circ} \mathrm{K}, 773^{\circ} \mathrm{K}$ and $1273^{\circ} \mathrm{K}$. (In the present paper. An example of the development of spinodal decomposition is visualized in Figs. 2a-2d for $T_{M C}=773^{\circ} \mathrm{K}$. Since we aim at collecting many varied spinodally-decomposed atomistic configurations, we run even at $T_{M C}=1273^{\circ} \mathrm{K}$, which is far above the experimental transition temperature of to $\gamma$-phase, which the potential is unable to reproduce. Although the numerical results at this $T_{M C}$ do not simulate any realistic binary alloys, we use this model material to produce varieties of Fe-Cr binary configuration. Strictly speaking, the Metropolis Monte Carlo method does not simulate the kinetics of spinodal decomposition because time is not defined, but in the present paper we are interested on snapshots along the pathway to a spinodally decomposed state. The snapshot data, i.e. locations and chemical identity of all the atoms, is recorded every 10 Monte Carlo steps (MCSs), where MCS is defined such that a swap attempt is performed on every atom per MCS.

To calculate $\mathrm{V}$ for each snapshot:

1. We decompose the whole simulation space into many small cubes composed of 100 atoms;

2. we measure the number of $\mathrm{Cr}$ atoms for all of these small cubes,

3. we count the number of small cubes that include $i(=0 \ldots 100) \mathrm{Cr}$ atoms; let's call this number as $S_{i}$, whcih gives a distribution parameterized by $\mathrm{Cr}$ concentration; 
Table 1: The atomic block used for the numerical study.

\begin{tabular}{ll}
\hline Name of parameter & value \\
\hline x-side length & $200 \AA$ \\
y-side length & $224 \AA$ \\
z-side length & $259 \AA$ \\
Total No. of atoms & $9.9 \times 10^{5}$ \\
Average Cr concentration & 25 at. $\%$ \\
\hline
\end{tabular}

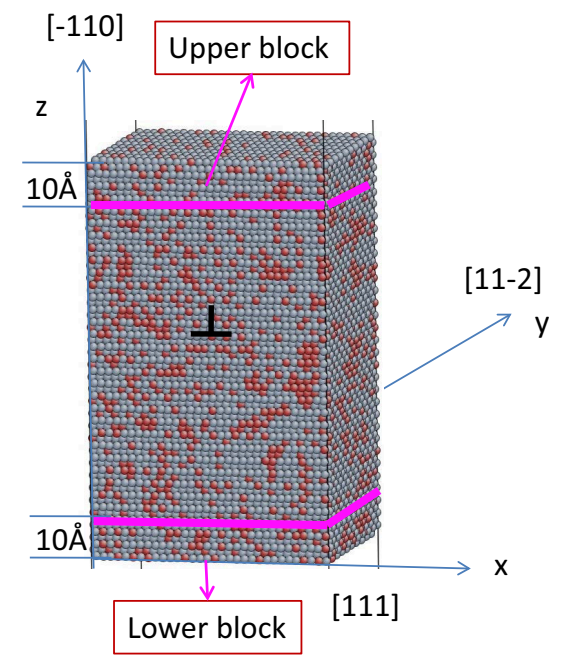

Figure 1: The atomic block used for the numerical studies. Grey and red spheres denote Fe and Cr atoms, respectively. 
(a) 0 MCS

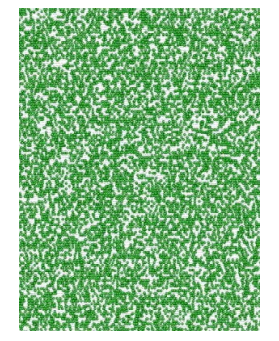

(c) 100 MCSs

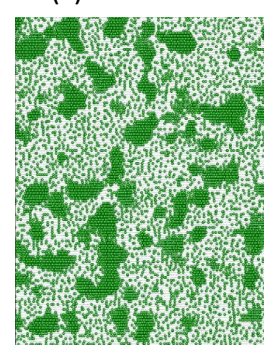

(b) 50 MCSs

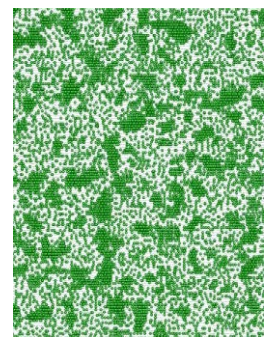

(d) 150 MCSs

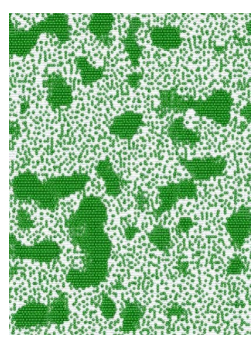

Figure 2: A Monte Carlo evolution of phase separation of Fe-Cr system at $T_{M C}=773^{\circ} \mathrm{K}$ : Only Cr atoms (green dots) are shown.

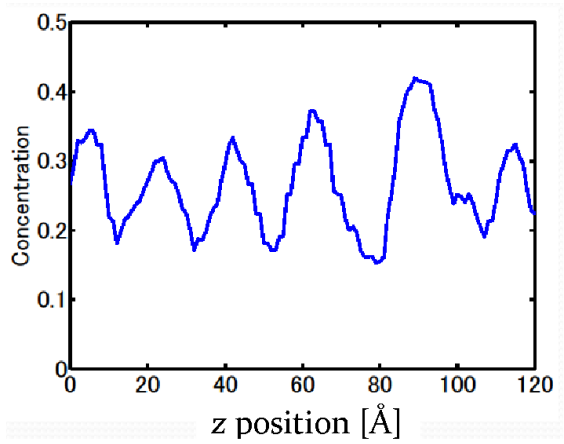

Figure 3: A typical example of a one-dimensional profile of $\mathrm{Cr}$ concentration for a spinodally modulated microstructure. 
4. $S_{i}$ is normalized as the integral of the whole distribution becomes unity; we call the normalized distribution as $O_{i}$;

The definition of the $\mathrm{V}$ is:

$$
V=\sum_{i=0}^{100}\left|O_{i}-B_{i}\right|,
$$

where $B_{i}$ is a binomial distribution defined by

$$
B_{i}=c_{0}\left(\begin{array}{c}
100 \\
i
\end{array}\right) p^{i}(1-p)^{100-i}
$$

where $p$ is the atomic concentration of $\mathrm{Cr} ; c_{0}$ is the factor to normalize the distribution so that $B_{i}$ agrees with $O_{i}$ when $\mathrm{Cr}$ atoms are located purely at random in the simulation space. It may be impossible to divide the simulation space as all the cubes include exactly 100 atoms, but distributions are rescaled so as to maintain their equivalence to the case when every cube contains exactly 100 atoms.

To characterize the modulation of $\mathrm{Cr}$ concentration, we use one-dimensional profiles of Cr concentration, obtained as follows: A small region in the atomic block, whose dimensions are $2.5 \mathrm{~nm} \times 2.5 \mathrm{~nm} \times 0.5 \mathrm{~nm}$, is defined, and atomic concentration of $\mathrm{Cr}$ atoms inside the region is measured at various $z$ positions while $(x, y)$ position are kept constant. An example of the one-dimensional Cr-concentration profile, $p(z)$, is given in Fig. 3. It appears to be random, but it is roughly a sinusoidal function that can be parameterized by an amplitude and a wavelength. To estimate these two parameters, its auto-correlation function, $c(z)$, is calculated from the fluctuation of the profile, $p(z)-<p(z)>$. By changing $(x, y)$ position, $\sim 50$ auto-correlation functions are calculated, and the mean autocorrelation function $<$ $c(z)>$ is obtained. To evaluate the amplitude of the fluctuation, some previous studies used a mean $\mathrm{Cr}$ concentration in $\alpha^{\prime}$-phase, but this method is confusing because it depends upon the $\mathrm{Cr}$ concentration threshold to determine the border between $\alpha$ and $\alpha$ '-phases. In this work we simply use standard deviations of the fluctuation of the profile. For the estimation of the wavelength, we measure the smallest positive $z$ value where $\langle c(z)>$ becomes zero, say $z_{1}$, and the wavelength is given by $4 z_{1}$.

According to [26] the SRO parameter for the $k$ th shell of a $\mathrm{Cr}$ atom is defined as

$$
\alpha_{C r}^{(k)}=1-\frac{Z_{F e}^{(k)}}{Z_{t o t}^{(k)}\left(1-c_{C r}\right)},
$$

where $Z_{F e}^{(k)}$ is the number of Fe atoms in the $k$ th shell, $Z_{t o t}^{(k)}$ the total number of atoms in the same shell, and $c_{C r}$ the overall $\mathrm{Cr}$ concentration. A coordination number-weighted average of the SRO parameter for the bcc lattice is defined as

$$
\beta=\frac{8 \alpha_{C r}^{(1)}+6 \alpha_{C r}^{(2)}}{14},
$$

and we exclusively use this definition for the SRO parameter in the following analyses. Many $\beta$ values are collected at various locations in the atomic block per snapshot, and their average, $\langle\beta\rangle$, is used as the final SRO parameter for the snapshot. 
Hardness is a property that depends in a complex way on individual and collective dislocation properties. Computer simulations can accurately address the onset of plasticity due to the initiation of dislocation motion, the yield stress. In this work we use yield stress as a property directly related to hardness and use indistinctly the expressions hardness and yield strength.

To measure the yield strength, we apply a method given in [27]. The atomic block is divided into three sub-blocks, say the upper, the middle, and the lower blocks, as shown in Fig. 1, then the whole atomic block is thermally equilibrated at $50^{\circ} \mathrm{K}$ and shear strain is applied in the $x$ direction by gradually shifting the upper block in the $x$ direction with the lower block completely fixed. Accordingly the pre-inserted edge dislocation moves in the $x$ direction.

The velocity of the shifting is $0.005 \AA / p s$, which for this sample size is equivalent to a strain rate of $2.08 \times 10^{-6} / \mathrm{ps}$. Note that this deformation rate is much larger than those imposed in actual experiments and would imply dislocation velocities in unrealistic high ranges. In these simulations however, we are only interested in the onset of plasticity, the yield stress (for the geometry we use, the yield stress measured is the critical resolved yield stress). This critical stress is also affected by the strain rate, as lower strain rates allow for thermal activated processes to also contribute to overcome the critical point. What our simulations explore is the stress-activated process of overcoming the obstacles generated by the spinodal decomposition, assuming that there is direct relationship between this stress and macroscopic measurements of hardening. From what is said above the simulation temperature for the study of dislocation motion is chosen to be low, namely $50^{\circ} \mathrm{K}$, in order to minimize thermal noise. The yield strength is measured as the maximum value of $\sigma_{x z}$, a component of stress tensor of the middle block. We confirmed that this value is reproduced in the different runs started from the same initial conditions at $50^{\circ} \mathrm{K}$; this indicates that thermal noise can be ignored at this temperature. Note that screw dislocations may be a cause of the hardening in this temperature range [28], but we were interested in the dependence of yield strength on microstructure at elevated temperatures, where only edge dislocations are relevant.

For this molecular dynamics simulation, we perform constant NVE integration (i.e. microcanonical molecular dynamics simulation) with a time step of $1 \mathrm{fs}$. Note that the temperature we use for studying dislocation dynamics is different from those used to decompose the alloy using VC-SGC Monte Carlo simulations.

\section{Results}

For each snapshot, we calculate the four characteristic parameters mentioned in the previous section; their evolution is shown in Figs. 4a-4d. One can recognize that all the parameters increase as the spinodal decomposition develops. Note that the evolution at $T_{M C}=773^{\circ} \mathrm{K}$ in Figs. $4 \mathrm{a}$ and $4 \mathrm{c}$ is almost identical to that with $1273^{\circ} \mathrm{K}$, while the evolution curves for wavelength and $\langle\beta\rangle$, shown in Figs. $4 \mathrm{~b}$ and $4 \mathrm{~d}$, are all distinct. This implies that the wavelength and $\langle\beta\rangle$ are sensitive to somewhat different microstructural properties. We come back to this matter in the next section. 
(a)

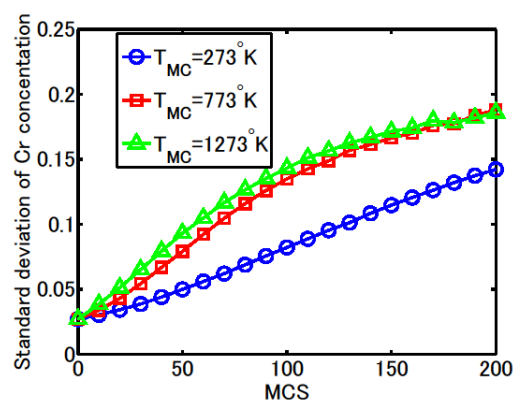

(c)

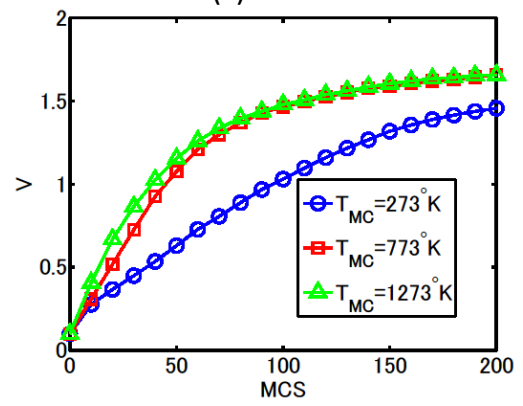

(b)

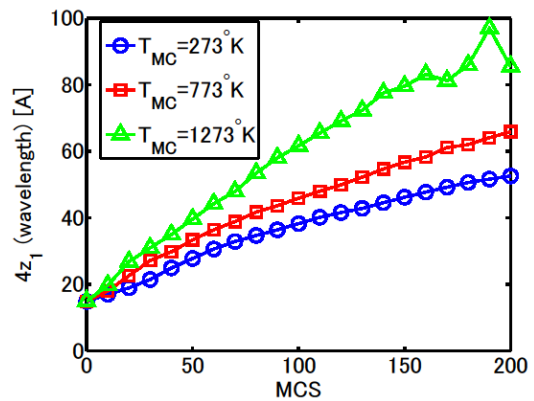

(d)

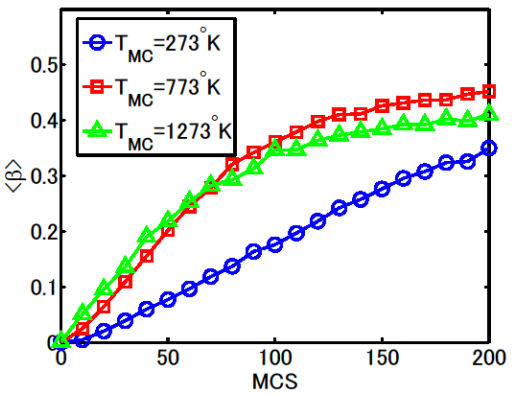

Figure 4: The development of the four characteristic parameters in the Monte Carlo simulation. 

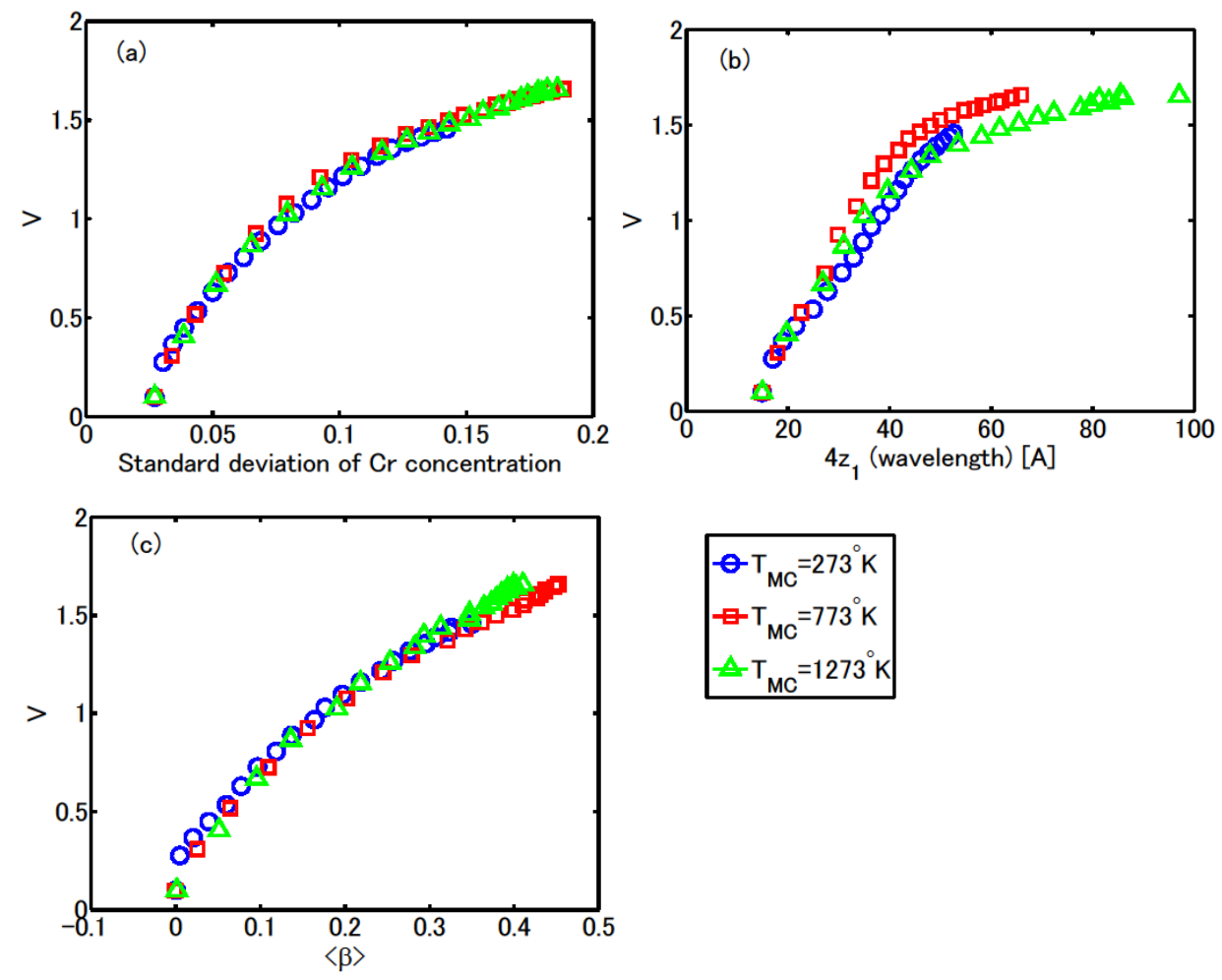

Figure 5: V vs. the three other characteristic parameters.

Fig. 5a shows the Vs as functions of the fluctuation amplitude of Cr-concentration profile shown in Fig. 3. The relation is constant over different microstructures obtained by decomposition at different Monte Carlo temperatures. With this inter-parameter analysis the meaning of the $\mathrm{V}$ became clearer, in that the $\mathrm{V}$ is approximately given by a unique nonlinear function of the fluctuation amplitude of the $\mathrm{Cr}$ concentration profile. Similar to this analysis, we also investigate the relationship between the $\mathrm{V}$ and the wavelength as shown in Fig. 5b. In this case the relationship is case-dependent, meaning that these two parameters are not directly related to each other.

Fig. 5c shows the relationship between $\mathrm{V}$ and SRO parameters. This relation is almost invariant over different Monte Carlo decomposition temperatures, but for the range of $V>\sim 1.3$ the case with $T_{M C}=1273^{\circ} \mathrm{K}$ departs from the others, indicating that these two parameters describe the development of spinodal decomposition in slightly different ways as suggested by comparing Figs. 4c and 4d.

Following the method described in the previous section, we conduct deformation molecular dynamics simulations to investigate the hardening as represented by the variations in yield stress. Typical stress-strain curves for different stages of the Monte Carlo decomposition microstructures are displayed in Fig. 6, which shows that the stress increases as strain 


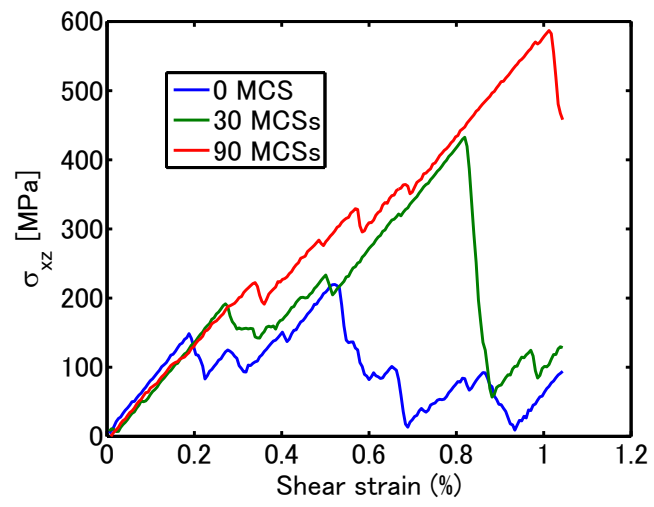

Figure 6: Stress vs. strain relationship during the shearing deformation: Snapshots with $T_{M C}=773^{\circ} \mathrm{K}$ were used.

increases, and then it suddenly drops at a critical point. This figure clearly indicates that more stress is necessary to yield as the phase separation proceeds further. To investigate the dislocation motion, we visualize the $x-y$ cross section of the atomic block at a $z$ position near the dislocation gliding plane, as seen in Fig. 7. These atomistic images are made from snapshots taken just before the $\sigma_{x z}$ became maximal, and one can see that the dislocation was pinned at $\alpha^{\prime}$-phase particles. As seen in Fig. 7a, the dislocation is almost straight in the early spinodal-decomposition stage when $\sigma_{x z}^{\max }$ is still small, while in the late stage, $\sigma_{x z}^{\max }$ is large and the dislocation is significantly bent (see Fig. 7b).

Three figures in Fig. 8 summarize the yield strength (i.e. $\sigma_{x z}^{\max }$ ) as functions of the three characteristic parameters, namely the wavelength, the $\mathrm{V}$ and SRO parameter. Each point in these figures corresponds to a snapshot of VC-SGC Monte Carlo decomposition simulations. To obtain better statistics we collect the hardening data from three sets of VC-SGC Monte Carlo simulations that are started from three different random atomistic configurations per $T_{M C}$ case, i.e. we conduct nine case studies in total.

There are common trends in all figures. First, the relationship is less stochastic for the early stage of phase decomposition, but random behavior emerges in the late stage. We believe that this is because fewer $\alpha$ '-phases are involved in the interaction with the dislocation at the late stage, and therefore the statistical error increases. Second, several points with $T_{M C}=273^{\circ} \mathrm{K}, 773^{\circ} \mathrm{K}$ deviate significantly upwards from general trend, but we do not interpret this facts as being relevant.

The relationship between $\sigma_{x z}^{\max }$ and the wavelength in Fig. 8a is linear and invariant with $T_{M C}$ at the early stage, but the values for $T_{M C}=1273^{\circ} \mathrm{K}$ clearly deviated downward from the linear relationship and became saturated in the late stage; the saturation against the wavelength is also observed in an experimental study of Vickers hardness at $773^{\circ} \mathrm{K}$ [29]. This implies that the case for $T_{M C}=1273^{\circ} \mathrm{K}$ reflects the realistic spinodal decomposed structures.

The relationship between the hardness and the $\mathrm{V}$ in Fig. 8b also shows a linear relationship as observed in past experimental studies. The relationship is independent of $T_{M C}$ at 
(a) $40 \mathrm{MCSs}, \sigma_{\mathrm{xz}}=394 \mathrm{MPa}$

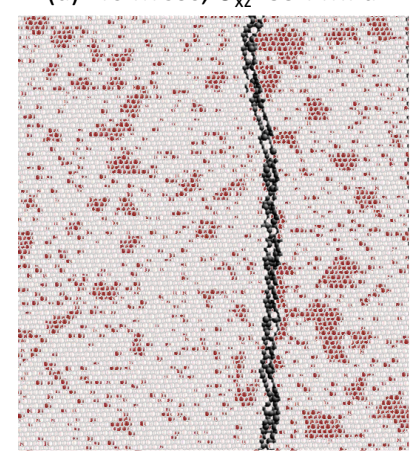

(b) 150 MCSs, $\sigma_{x z}=780 \mathrm{MPa}$

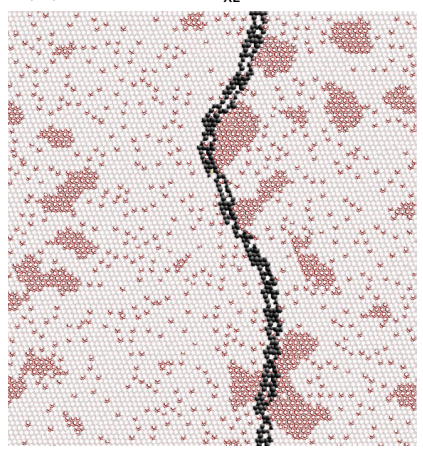

Figure 7: Atomistic view of an edge dislocation interacting $\alpha^{\prime}$-phases with $T_{M C}=773^{\circ} K$ : The dislocations are shown as a chain of atoms whose coordination number is not 14; Views were taken when $\sigma_{x z}$ became almost maximal. 

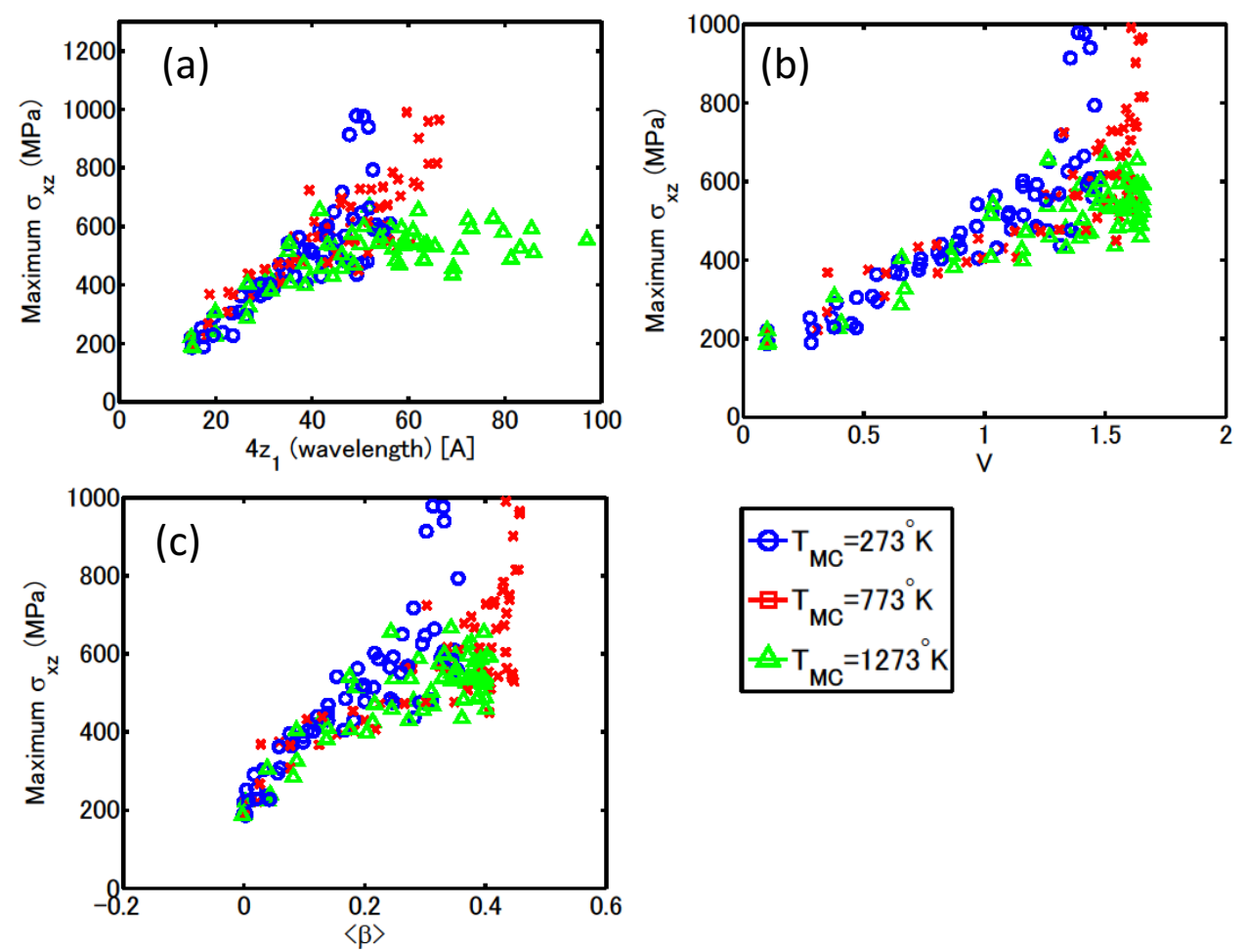

Figure 8: The maximum value of $\sigma_{x z}$ vs. the characteristic parameters.

the early stage, but it becomes dependent upon $T_{M C}$ as $\mathrm{V}$ grows, that is, the yield strength is greater for the lower $T_{M C}$ as similarly observed in the yield strength - wavelength relationship of Fig. 8a. The results here suggest that the experimentally observed linear relationship is not universal for the different stages of Fe-25Cr phase separation, especially in the late stage of spinodal decomposition.

General trends of the hardness-SRO relationship shown in Fig. 8c are similar to those between the hardness and the $\mathrm{V}$, although there were a couple of different features that must not be ignored: The values for different $T_{M C}$ s now became overlapped except for the extreme points mentioned above; the difference from Fig. 8b is subtle, but recognizable. This means that the relationship is universal over the different $T_{M C} \mathrm{~s}$ and that SRO parameter captures better the microstructural property with respect to hardening. The other point of difference is a nonlinearity at the early stage.

\section{Discussion}

As shown above, the SRO parameter captures better the microstructural properties involved with hardening (as measured by yield strength), implying that hardness depends 
more on atomic-scale properties than nanoscale configurations. This suggests that the relation between $\sigma_{x z}^{\max }$ and the wavelength measured through auto-correlation function, which is an above-nanoscale property, in Fig. 8a is less physically significant, and that hardening cannot not be directly associated with the increase of the wavelength. Note that $\sigma_{x z}^{\max }$ becomes saturated as seen in Fig. 8a; this also supports the idea that the wavelength has little relevance to the hardening, and is not a good indicator of the development of hardening.

The saturation of hardening in Fig. 8a also indicates that some recovery phenomena occur in parallel with the increase in wavelength at $T_{M C}=1273^{\circ} \mathrm{K}$, as explained below. From Fig. 4b, one can recognize that the wavelength in the late stage at this $T_{M C}$ is larger than that at $773^{\circ} \mathrm{K}$; this fact indicates that larger $\alpha^{\prime}$-phases are formed at $1273^{\circ} \mathrm{K}$. On the other hand, the SRO parameter in the late stage at $1273^{\circ} \mathrm{K}$ is smaller than that at $773^{\circ} \mathrm{K}$ (Fig. 4d); this indicates that Cr's spatial distribution is less ordered at $1273^{\circ} \mathrm{K}$. These two facts suggest that large $\alpha^{\prime}$-phases formed at $1273^{\circ} \mathrm{K}$ have more mixed composition than smaller $\alpha^{\prime}$-phases at the lower $T_{M C}$. The mixing is surely caused by high Monte Carlo temperature, and we believe that this mixing is the cause of the saturated hardening.

Such recovery phenomena were observed in some past experimental studies; for example Li et al. [30] reports the recovery of mechanical properties through annealing at $823^{\circ} \mathrm{K}$. In addition, Fujii et al. [17] also reports that hardness decreased after ion irradiation, suggesting that the mechanical property is recovered. Their atom probe tomography indicates that the image of $\alpha^{\prime}$-phase become less sharp and the $\alpha-\alpha^{\prime}$ boundaries become blunt after the ion irradiation, indicating athermal mixing. Note that some experimental studies also indicate that neutron irradiation enhances the spinodal decomposition and the hardening [18, 31]; we think that in these cases, irradiation rates are too low to cause the athermal mixing that leads to recovery.

Note that $\mathrm{V}$, as seen in Fig. 8b, has better universality than the wavelength as an indicator of hardening. However, $\mathrm{V}$ is not sensitive to some microstructural properties which SRO is sensitive to, as mentioned in the previous section, see Figs. 4c and 4d; this is the reason why $\mathrm{V}$ and $\langle\beta\rangle$ relationship in Fig. 5c was dependent on $T_{M C}$. Since this temperature dependence occurs only in the late stage of the Monte Carlo decomposition, we believe that $\mathrm{V}$ is less sensitive to the thermal mixing mentioned above, and this must be the reason for the upper limit of universal relationship between yield strength and V in Fig. 8b. Consequently, the performance of $\mathrm{V}$ as an empirical indicator of hardening has a limitation on its universality, as it does not properly captures short-range atomistic configurations that influence hardening. A disadvantage of SRO parameter as a hardness indicator is that the relation to yield strength is nonlinear, while V's relation to yield strength is linear.

This upper limit of $\mathrm{V}$ might be recognized as the early stage of transition from the spinodally modulated structure to the dispersed independent $\alpha$ '-phase particles, as shown in Fig. 2. If each particle becomes isolated enough from others, the hardness might eventually been given by a model for precipitation hardening, such as the well-known Russel-Brown model [32]. 


\section{Conclusions}

We investigated the statistical indicators of spinodally decomposed Fe-25Cr binary alloys by using Monte Carlo simulations to induce spinodal decomposition, followed by molecular dynamics simulations to induce plasticity, in order to study the relationships between hardening of these alloys (as measured by yield strength) and these indicators. We confirmed that $\mathrm{V}$ is uniquely determined by a nonlinear function of the amplitude of the modulation wave of $\mathrm{Cr}$ concentration, and the physical meaning of this parameter becomes clearer. The parameter $\mathrm{V}$ is linearly related to the increase in hardness as observed in the experiments, and this relation is invariant with the Monte Carlo temperature used for the decomposition; its usefulness as a indicator of hardening is then supported. At the same time we found that such usefulness is limited: Above an upper limit of $\mathrm{V}(\sim 1.3)$, i.e. at the late stage of the spinodal decomposition, the hardness- $V$ relation became nonlinear and Monte-Carlodecomposition temperature dependent. The upper limit seems to be caused by a recovery phenomenon induced by thermal mixing.

Short-range order (SRO) parameter was also examined as such an indicator, and its relation to yield strength was almost independent of the Monte Carlo decomposition temperature for the full range of the phase-separation development. This suggests that short-range atomistic configuration is changed by thermal mixing and influences hardness, and that such a microscopic effect is not sensitive to $\mathrm{V}$. In conclusion, the SRO parameter could become a better empirical indicator for the phenomenon of hardening. To be fair, we would like to mention that the relation between SRO parameter and yield strength is not linear, so the SRO parameter has a demerit as a hardening indicator as well as a merit.

In future studies, we will collect a greater variety of experimental and computational data and formalize their statistical analyses into a universal empirical equation for hardening, which would be useful for monitoring the development of hardening in Fe-Cr alloys.

\section{Acknowledgements}

The first author (T. Suzudo) thanks to T. Tsuru of Japan Atomic Energy Agency for helpful discussion on molecular dynamics simulations. This work include the result of "Research and development on degradation prediction of structural materials in nuclear reactors based on microstructural damage mechanisms" entrusted to Tohoku university by Education, Culture, Sports, Science and Technology of Japan (MEXT). A. Caro acknowledges support from the Center for Materials under Irradiation and Mechanical Extremes, a DOEOBES Energy Frontier Research Center.

\section{References}

[1] Miller, M., Hyde, J., Hetherington, M., Cerezo, A., Smith, G., Elliott, C.. Acta Metallurgica et Materialia 1995;43(9):3385-3401.

[2] Hyde, J., Miller, M., Hetherington, M., Cerezo, A., Smith, G., Elliott, C.. Acta metallurgica et materialia 1995;43(9):3403-3413.

[3] Hyde, J., Miller, M., Hetherington, M., Cerezo, A., Smith, G., Elliott, C.. Acta metallurgica et materialia 1995;43(9):3415-3426. 
[4] Cortie, M., Pollak, H.. Materials Science and Engineering: A 1995;199(2):153-163.

[5] Lo, K., Shek, C., Lai, J.. Materials Science and Engineering: R: Reports 2009;65(4):39-104.

[6] Klueh, R., Nelson, A.. Journal of Nuclear Materials 2007;371(1):37-52.

[7] Cahn, J.W.. Acta Metallurgica 1963;11(12):1275-1282.

[8] Kato, M., Mori, T., Schwartz, L.H.. Acta Metallurgica 1980;28(3):285-290.

[9] Lagneborg, R.. Acta Metallurgica 1967;15(11):1737-1745.

[10] Wagner, R.. Czechoslovak Journal of Physics 1981;31(2):198-208.

[11] Terentyev, D., Bonny, G., Malerba, L.. Acta Materialia 2008;56(13):3229-3235.

[12] Williams, R.. Acta Metallurgica 1957;5(7):385-392.

[13] Bonny, G., Terentyev, D., Malerba, L.. Journal of Nuclear Materials 2009;385(2):278-283.

[14] Blavette, D., Grancher, G., Bostel, A.. Le Journal de Physique Colloques 1988;49(C6):C6-433.

[15] Pareige, C., Novy, S., Saillet, S., Pareige, P.. Journal of Nuclear Materials 2011;411(1):90-96.

[16] Takeuchi, T., Kakubo, Y., Matsukawa, Y., Nozawa, Y., Nagai, Y., Nishiyama, Y., et al. Journal of Nuclear Materials 2013;443(1):266-273.

[17] Fujii, K., Fukuya, K.. Journal of Nuclear Materials 2013;440(1):612-616.

[18] Takeuchi, T., Kakubo, Y., Matsukawa, Y., Nozawa, Y., Toyama, T., Nagai, Y., et al. Journal of Nuclear Materials 2014;

[19] Cowley, J.. Physical Review 1950;77:669-675.

[20] Olsson, P., Wallenius, J., Domain, C., Nordlund, K., Malerba, L., Physical Review B 2005;72:214119.

[21] Caro, A., Crowson, D., Caro, M.. Physical review letters 2005;95(7):075702.

[22] Stukowski, A., Sadigh, B., Erhart, P., Caro, A.. Modelling and Simulation in Materials Science and Engineering 2009;17(7):075005.

[23] Bonny, G., Pacianot, R.C., Malerba, L., Caro, A., Olsson, P., Lavrentiev, M.Yu., Journal of Nuclear Materials 2009;385(2):268-277.

[24] Plimpton, S., et al. Journal of Computational Physics 1995;117(1):1-19.

[25] Sadigh, B., Erhart, P., Stukowski, A., Caro, A., Martinez, E., Zepeda-Ruiz, L.. Physical Review B 2012;85(18):184203.

[26] Erhart, P., Caro, A., de Caro, M.S., Sadigh, B.. Physical Review B 2008;77(13):134206.

[27] Osetsky, Y.N., Bacon, D., Mohles, V.. Philosophical Magazine 2003;83(31-34):3623-3641.

[28] Gilbert, M., Queyreau, S., Marian, J.. Physical Review B 2011;84(17):174103.

[29] Soriano-Vargas, O., Avila-Davila, E.O., Lopez-Hirata, V.M., Cayetano-Castro, N., GonzalezVelazquez, J.L.. Materials Science and Engineering: A 2010;527(12):2910-2914.

[30] Li, S., Zhang, H., Wang, Y., Li, S., Zheng, K., Xue, F., et al. Materials Science and Engineering: A 2013;564:85-91.

[31] Miller, M., Stoller, R., Russell, K.. Journal of nuclear materials 1996;230(3):219-225.

[32] Russell, K.C., Brown, L.. Acta Metallurgica 1972;20(7):969-974. 